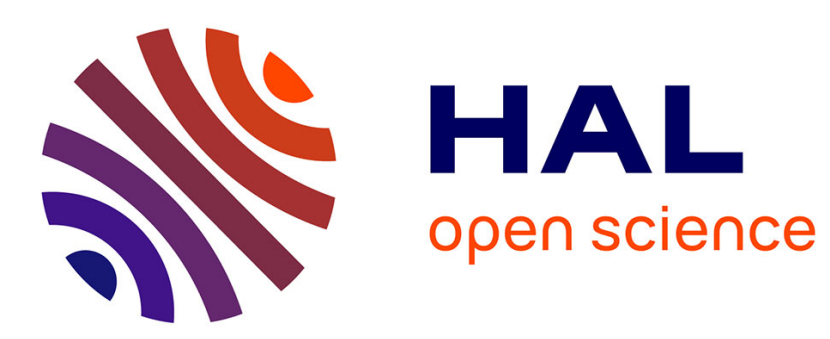

\title{
Simulation of a liquid-vapour compressible flow by a Lattice Boltzmann Method
}

Philippe Helluy, Olivier Hurisse, Lucie Quibel

\section{To cite this version:}

Philippe Helluy, Olivier Hurisse, Lucie Quibel. Simulation of a liquid-vapour compressible flow by a Lattice Boltzmann Method. Finite Volumes for Complex Applications IX - Methods, Theoretical Aspects, Examples. FVCA 2020., 2020, 978-3-030-43650-6. 10.1007/978-3-030-43651-3_63 . hal02451368

\section{HAL Id: hal-02451368 \\ https://hal.science/hal-02451368}

Submitted on 23 Jan 2020

HAL is a multi-disciplinary open access archive for the deposit and dissemination of scientific research documents, whether they are published or not. The documents may come from teaching and research institutions in France or abroad, or from public or private research centers.
L'archive ouverte pluridisciplinaire HAL, est destinée au dépôt et à la diffusion de documents scientifiques de niveau recherche, publiés ou non, émanant des établissements d'enseignement et de recherche français ou étrangers, des laboratoires publics ou privés. 


\title{
Simulation of a liquid-vapour compressible flow by a Lattice Boltzmann Method
}

\author{
Philippe Helluy, Olivier Hurisse and Lucie Quibel
}

\begin{abstract}
This work is devoted to the numerical resolution of a compressible three5 phase flow with phase transition by a Lattice-Boltzmann Method (LBM). The flow presents complex features and large variations of physical quantities. The LBM is a robust numerical method that is entropy stable and that can be extended to second order accuracy without additional numerical cost. We present preliminary numerical results, which confirm its competitiveness compared to other Finite Volume meth-
\end{abstract} 10 ods.

Key words: Lattice Boltzmann Method, compressible flow, phase transition.

MSC (2010): 35Q79, 76M12, 76M28

\section{Introduction}

In this work, we are interested in the numerical resolution of a hyperbolic system arising in thermohydraulics. The objective is to compute a three-phase flow made of liquid water, vapour and an inert gas (such as air, for instance). Because of the envisaged range of pressure and temperature, there can be phase transition between the liquid and its vapour.

The Equation Of State (EOS) is complex and presents large variations of the thermodynamical parameters. It can be obtained from physical experiments and tabulations. It generally leads to very costly numerical methods, where most of the time

Philippe Helluy

IRMA UMR 7501, Inria Tonus

Université de Strasbourg, France

e-mail: philippe.helluy@unistra.fr

Olivier Hurisse, Lucie Quibel

EDF R\&D, 6 quai Watier, 78400 Chatou, France.

e-mail: olivier.hurisse@edf.fr, lucie.quibel@edf.fr 
is spent in the evaluation of the EOS. In addition, if because of the approximation the EOS does not satisfy some convexity properties, the resulting system of conservation laws may not be hyperbolic and thus unstable. Here we use a simplified pressure law obtained from an entropy optimization procedure. The pressure law was first described in [1]. By construction, it ensures a convex hyperbolic domain and thus stability of some classical Finite Volume (FV) schemes such as Godunovtype schemes [7] or the Bouchut kinetic scheme [2].

The standard FV method is only first order. Its accuracy can be improved by slope reconstruction/limitation techniques. But this induces a cost and a more difficult parallelization because the computation stencil is enlarged.

In this work, we replace the FV scheme by a Lattice Boltzmann Method (LBM). The LBM is based on an abstract kinetic representation of the hyperbolic system. Then the scheme is a succession of free transport steps solved by an exact characteristic shift and relaxation operations that are local to the cell. This makes the LBM very efficient and easy to parallelize. In addition, by simply changing the relaxation parameter, it is possible to adjust the numerical viscosity of the LBM and to achieve second order with no additional cost.

We apply the whole approach for computing a vapour explosion test case.

\section{Kinetic approximation of conservation laws}

\subsection{Vectorial kinetic approximation with over-relaxation}

In this work, we are interested in the numerical resolution of a hyperbolic system arising in thermohydraulics. The vector of unknown is denoted $\mathbf{u}(x, t) \in \mathbb{R}^{m}$. The system has the general form

$$
\partial_{t} \mathbf{u}+\partial_{x} \mathbf{f}(\mathbf{u})=0 .
$$

The flux $\mathbf{f}$ is a smooth function $\mathbb{R}^{m} \mapsto \mathbb{R}^{m}$ satisfying the hyperbolicity property: its jacobian matrix $\mathbf{f}^{\prime}(\mathbf{u})$ is diagonalizable with real eigenvalues for all $\mathbf{u}$ in the hyperbolicity domain $\mathscr{C}$, which is assumed to be convex. The relaxation approach, introduced by Jin and Xin [9], consists in replacing (1) by an extended system of the form

$$
\begin{aligned}
\partial_{t} \mathbf{u}+\partial_{x} \mathbf{z} & =0, \\
\partial_{t} \mathbf{z}+\lambda^{2} \partial_{x} \mathbf{u} & =\mu .
\end{aligned}
$$

45 The speed $\lambda$ is a positive constant. The new vector $\mathbf{z}$ is called the approximated flux. The source term $\mu$ is designed in such a way that $\mathbf{z} \simeq \mathbf{f}(\mathbf{u})$. We introduce a time step $\Delta t>0$ and the Dirac comb: 


$$
\Psi(t)=\sum_{i \in \mathbb{Z}} \delta(t-i \Delta t) .
$$

The source term $\mu$ is then defined by

$$
\mu(x, t)=\Omega \Psi(t)\left(\mathbf{f}(\mathbf{u}(x, t))-\mathbf{z}\left(x, t^{-}\right)\right), \quad \mathbf{I} \leq \boldsymbol{\Omega} \leq \mathbf{2} \mathbf{I} .
$$

In the more general case, $\Omega$ is a matrix called the relaxation matrix. Inequalities on matrices have to be understood, as usual, in the sense of the associated quadratic forms. From the distribution theory, we see that at time $t=i \Delta t, \mathbf{z}$ is discontinuous: $\mathbf{z}\left(x, t^{+}\right) \neq \mathbf{z}\left(x, t^{-}\right)$, and

$$
\mathbf{z}\left(x, t^{+}\right)=\Omega \mathbf{f}(\mathbf{u}(x, t))+(\mathbf{I}-\Omega) \mathbf{z}\left(x, t^{-}\right) .
$$

If the relaxation matrix $\Omega=\mathbf{I}$, we recover in this way the classical first order splitting Jin-Xin algorithm, where $\mathbf{z}=\mathbf{f}(\mathbf{u})$ at the end of each time step. The overrelaxation corresponds to $\Omega=2 \mathbf{I}$. It can be proved that the resulting scheme is a second order $O\left(\Delta t^{2}\right)$ approximation of (1). See [3,5], for instance, and included references.

We can diagonalize the linear hyperbolic operator arising from the left-hand side of (2)-(3). In this way, we obtain a kinetic interpretation of the Jin-Xin approximation. For this, we consider the change of variables

$$
\begin{aligned}
& \mathbf{k}^{+}=\frac{\mathbf{u}}{2}+\frac{\mathbf{z}}{2 \lambda}, \quad \mathbf{k}^{-}=\frac{\mathbf{u}}{2}-\frac{\mathbf{z}}{2 \lambda} . \\
& \mathbf{u}=\mathbf{k}^{+}+\mathbf{k}^{-}, \quad \mathbf{z}=\lambda \mathbf{k}^{+}-\lambda \mathbf{k}^{-} .
\end{aligned}
$$

Then we get

$$
\partial_{t} \mathbf{k}^{+}+\lambda \partial_{x} \mathbf{k}^{+}=\mathbf{r}^{+}, \quad \partial_{t} \mathbf{k}^{-}-\lambda \partial_{x} \mathbf{k}^{-}=\mathbf{r}^{-},
$$

where

$$
\mathbf{r}^{ \pm}(x, t)=\Omega \Psi(t)\left(\mathbf{k}^{e q, \pm}\left(\mathbf{u}\left(x, t^{-}\right)\right)-\mathbf{k}^{ \pm}\left(x, t^{-}\right)\right),
$$

and the "Maxwellian" states $\mathbf{k}^{e q, \pm}$ are given by

$$
\mathbf{k}^{e q, \pm}(\mathbf{u})=\frac{\mathbf{u}}{2} \pm \frac{\mathbf{f}(\mathbf{u})}{2 \lambda} .
$$

In other words, from these calculations, we see that most of the time, the kinetic variables $\mathbf{k}^{+}$and $\mathbf{k}^{-}$satisfy free transport equations at velocity $\pm \lambda$, with relaxation to equilibrium at each time step.

\subsection{Equivalent equation}

The equivalent equation allows to better understand the effect of the relaxation ma- 
Theorem 1 If the relaxation matrix satisfies $\mathbf{I}<\Omega<2 \mathbf{I}$ and if $\mathbf{y}=0$ at the initial time, then, up to second order terms in $O\left(\Delta t^{2}\right)$, $\mathbf{u}$ is a solution of the following system of conservation laws

$$
\partial_{t} \mathbf{u}+\partial_{x} \mathbf{f}(\mathbf{u})=\Delta t \partial_{x}\left(\left(\Omega^{-1}-\frac{1}{2} \mathbf{I}\right)\left(\lambda^{2} \mathbf{I}-\mathbf{f}^{\prime}(\mathbf{u})^{2}\right) \partial_{x} \mathbf{u}\right)+O\left(\Delta t^{2}\right) .
$$

Remark 1 The proof is based on standard Taylor expansions. For a rigorous formulation and proof, we refer to [4]. The approach is classical in the analysis of the Lattice Boltzmann Method (LBM). See also for instance [6, 10, 5].

Remark 2 The above analysis allows to recover formally the so-called sub-characteristic condition. Assuming that $\mathbf{I}<\Omega<2 \mathbf{I}$, the second order ("viscous") terms have the good sign, which ensures stability of the model, if the following matrix is positive:

$$
\mathbf{V}(\mathbf{u})=\lambda^{2} \mathbf{I}-\mathbf{f}^{\prime}(\mathbf{u})^{2}>0 .
$$

\section{Numerical methods}

Our objective is to design a specific Lattice Boltzmann Method (LBM) for approximating three-phase flow. For comparison, we need a classical finite volume method, which we describe now.

\subsection{Finite volume method}

The finite volume scheme (FV scheme in the sequel) is constructed for approximating the solutions of (1). We denote by $\Delta x$ the space step and by $\Delta t$ the time step. We assume that the space step and the time step are related by a Courant-FriedrichsLewy (CFL) relation $\Delta t=\beta \frac{\Delta x}{\lambda}$, where $\beta>0$ is the CFL number. We use the same velocity $\lambda$ in the FV and LBM methods for defining the CFL number. Because of the sub-characteristic condition (5), $\lambda$ is larger than the wave speeds of (1). We thus expect that the FV scheme will be stable at least for $\beta<1$.

We look for an approximation

$$
\mathbf{u}_{i}^{n} \simeq \frac{1}{\Delta x} \int_{x_{i-1 / 2}}^{x_{i+1 / 2}} \mathbf{u}\left(x, t_{n}\right) d x \simeq \mathbf{u}\left(x_{i}, t_{n}\right), \quad x_{i}=i \Delta x, \quad t_{n}=n \Delta t .
$$

We consider the FV scheme

$$
\frac{\mathbf{u}_{i}^{n+1}-\mathbf{u}_{i}^{n}}{\Delta t}+\frac{\mathbf{f}\left(\mathbf{u}_{i}^{n}, \mathbf{u}_{i+1}^{n}\right)-\mathbf{f}\left(\mathbf{u}_{i-1}^{n}, \mathbf{u}_{i}^{n}\right)}{\Delta x}=0 .
$$

The numerical flux $\mathbf{f}(\cdot, \cdot)$ is the Rusanov flux given by 


$$
\mathbf{f}(\mathbf{u}, \mathbf{v})=\frac{\mathbf{f}(\mathbf{u})+\mathbf{f}(\mathbf{v})}{2}-\frac{\max \left(\rho\left(\mathbf{f}^{\prime}(\mathbf{u})\right), \rho\left(\mathbf{f}^{\prime}(\mathbf{v})\right)\right)}{2}(\mathbf{v}-\mathbf{u}),
$$

where $\rho(\mathbf{M})$ is the spectral radius of matrix $\mathbf{M}$.

\subsection{Lattice Boltzmann Method (LBM)}

In the LBM scheme, we assume that the CFL number $\beta=1$. This allows to solve the free transport steps exactly. More precisely, if we also denote by $\mathbf{u}_{i}^{n}, \mathbf{z}_{i}^{n}, \mathbf{k}_{i}^{ \pm, n}$ the approximation of $\mathbf{u}, \mathbf{z}$ and $\mathbf{k}^{ \pm}$at points $x_{i}$ and time $t_{n}$, the transport step is given by simple shift operations, which solve the free transport equations (4) exactly

$$
\mathbf{k}_{i}^{-, n+1-}=\mathbf{k}_{i+1}^{-, n}, \quad \mathbf{k}_{i}^{+, n+1-}=\mathbf{k}_{i-1}^{+, n} .
$$

Then, one takes

$$
\mathbf{u}_{i}^{n+1}=\mathbf{k}_{i}^{-, n+1-}+\mathbf{k}_{i}^{+, n+1-}, \quad \mathbf{z}_{i}^{n+1-}=-\lambda \mathbf{k}_{i}^{-, n+1-}+\lambda \mathbf{k}_{i}^{+, n+1-} .
$$

The relaxation step is then

$$
\mathbf{z}_{i}^{n+1}=\mathbf{z}_{i}^{n+1-}+\Omega\left(\mathbf{u}_{i}^{n+1}\right)\left(\mathbf{f}\left(\mathbf{u}_{i}^{n+1}\right)-\mathbf{z}_{i}^{n+1-}\right) .
$$

\section{Application to a three-phase flows}

We wish to apply the above theory to a compressible three-phase flow model (two gases and a liquid). Because of strong variations in pressure and temperature, the liquid will undergo phase transition, which requires a proper mathematical model. The unknowns are the density $\rho$, the velocity $u$, the pressure $p$, the internal energy $e$ and the mass fraction of the inert gas $\varphi=\varphi_{3}$. The total energy $E$ is the sum of the internal energy and the kinetic energy: $E=\rho e+\frac{1}{2} \rho u^{2}$. The pressure Equation Of State (EOS) is of the form $p=p(\rho, e, \varphi)$. The three-phase flow model is a system of conservation laws of the form (1) with

$$
\mathbf{u}=(\rho, \rho u, \rho E, \rho \varphi)^{\top}, \quad \mathbf{f}(\mathbf{u})=\left(\rho u, \rho u^{2}+p,(\rho E+p) u, \rho u \varphi\right)^{\top} .
$$

Now we sketch the practical construction of the three-phase pressure law. This construction has to be done with care in order to ensure that the hyperbolicity domain $\mathscr{C}$ is convex. The general principles are mainly given in $[8,1]$. We consider

a mixture of three phases (1), (2) and (3) representing the vapour, the liquid and the non-condensable gas (air), respectively. The liquid is not miscible with the two others, while the vapour and the gas are miscible. We only admit phase transition between vapour (1) and liquid (2). Each phase obeys a stiffened gas Equation Of State (EOS), where the entropy function is defined by 


$$
s_{i}\left(\tau_{i}, e_{i}\right)=C_{i} \ln \left(\left(e_{i}-Q_{i}-\pi_{i} \tau_{i}\right) \tau_{i}^{\gamma_{i}-1}\right)+s_{i}^{0} \quad i=1,2,3 .
$$
$\pi_{i}$ is the reference pressure and $s_{i}^{0}$ the reference entropy. The specific energy is noted $e_{i}$ and the specific volume $\tau_{i}$ is the inverse of the density $1 / \rho_{i}$. Some possible parameters are given in Table 1 .

The mass fractions of the phases are noted $\varphi_{i}$, the volume fractions, $\alpha_{i}$ and the energy fractions, $\zeta_{i}$. The phase specific volumes $\tau_{i}$ and energies $e_{i}$ are related to the mixture specific volume $\tau$ and energy $e$ by

$$
\tau_{i}=\frac{\alpha_{i}}{\varphi_{i}} \tau, \quad e_{i}=\frac{\zeta_{i}}{\varphi_{i}} e
$$

The mass fraction $\varphi_{3}$ of the inert gas is supposed to be fixed and given. We thus introduce the vector of the unknown fractions $Y=\left(\varphi_{1}, \varphi_{2}, \alpha_{1}, \alpha_{2}, \alpha_{3}, \zeta_{1}, \zeta_{2}, \zeta_{3}\right)$. The unknown fractions satisfy the following constraints

$$
Y \in Q:=[0,1]^{8} \cap\left\{\alpha_{1}=\alpha_{3}, \alpha_{1}+\alpha_{2}=1, \varphi_{1}+\varphi_{2}+\varphi_{3}=1, \zeta_{1}+\zeta_{2}+\zeta_{3}=1\right\} .
$$

These constraints are justified by the fact that the two gases are perfectly miscible (Dalton's law) and that the liquid and the gases are non-miscible. The mixture entropy is then given by a convex optimization problem:

$$
s\left(\tau, e, \varphi_{3}\right)=\max _{Y \in Q} \sum_{i=1}^{3} \varphi_{i} s_{i}\left(\frac{\alpha_{i}}{\varphi_{i}} \tau, \frac{\zeta_{i}}{\varphi_{i}} e\right) .
$$

Once the optimization problem is solved, the temperature $T$ and the pressure $p$ of the mixture are then given by

$$
T=1 / \frac{\partial s}{\partial e} \quad p=T \frac{\partial s}{\partial \tau}
$$

We have no place to detail the computations. We refer to [1]. The major advantage of the above construction is that it ensures that the hyperbolicity domain is convex.

\section{Vapour explosion test}

We consider a test case relevant for thermohydraulics. This is quite a realistic modelling of a sudden depressurization of a heated liquid in a pipe. The left (L) part of the pipe is filled with pressurized heated water. The right $(\mathrm{R})$ part of the pipe is filled with air at ambient temperature and pressure. The numerical parameters are summed up in Table 1.

At time $t=0$, the liquid-air separation is removed. We plot several physical quantities at time $t=1.2 \mathrm{~ms}$. We observe a complex wave structure. From left to right: 


\begin{tabular}{|c|c|c|c|}
\hline param. & vapour (1) & liquid (2) & gas (3) \\
\hline \hline$\gamma_{i}$ & 1.3 & 3 & 1.4 \\
\hline$\pi_{i}(\mathrm{~Pa})$ & 0 & $8533 \times 10^{5}$ & 0 \\
\hline$C_{i}\left({\left.\mathrm{~J} . \mathrm{kg}^{-1} \cdot \mathrm{K}^{-1}\right)}^{-1}\right.$ & 1615.38 & 1400 & 719.28 \\
\hline$Q_{i}\left({\left.\mathrm{~J} . \mathrm{kg}^{-1}\right)}^{0}\right.$ & $1.892 \times 10^{6}$ & $-1.1148 \times 10^{6}$ & 0 \\
\hline$s_{i}^{0}$ & 583.46 & 16658.99 & 263.62 \\
\hline
\end{tabular}

\begin{tabular}{|c|c|c|}
\hline & liquid (L) & air (R) \\
\hline \hline$\rho$ & 554.09 & 1.186245 \\
\hline$e$ & 1161999.729 & 210749.040 \\
\hline$\varphi$ & $10^{-6}$ & $1-10^{-6}$ \\
\hline
\end{tabular}

Table 1 Left: physical parameters for the three phases. Right:initial data for the vapor explosion test case.

the liquid, a contact wave, and finally a shock wave running into the air. Let us remark the presence of a non-standard split wave made of two simple waves. This is a typical feature of Riemann problems with non-convex equations of state arising from phase transition problematics.
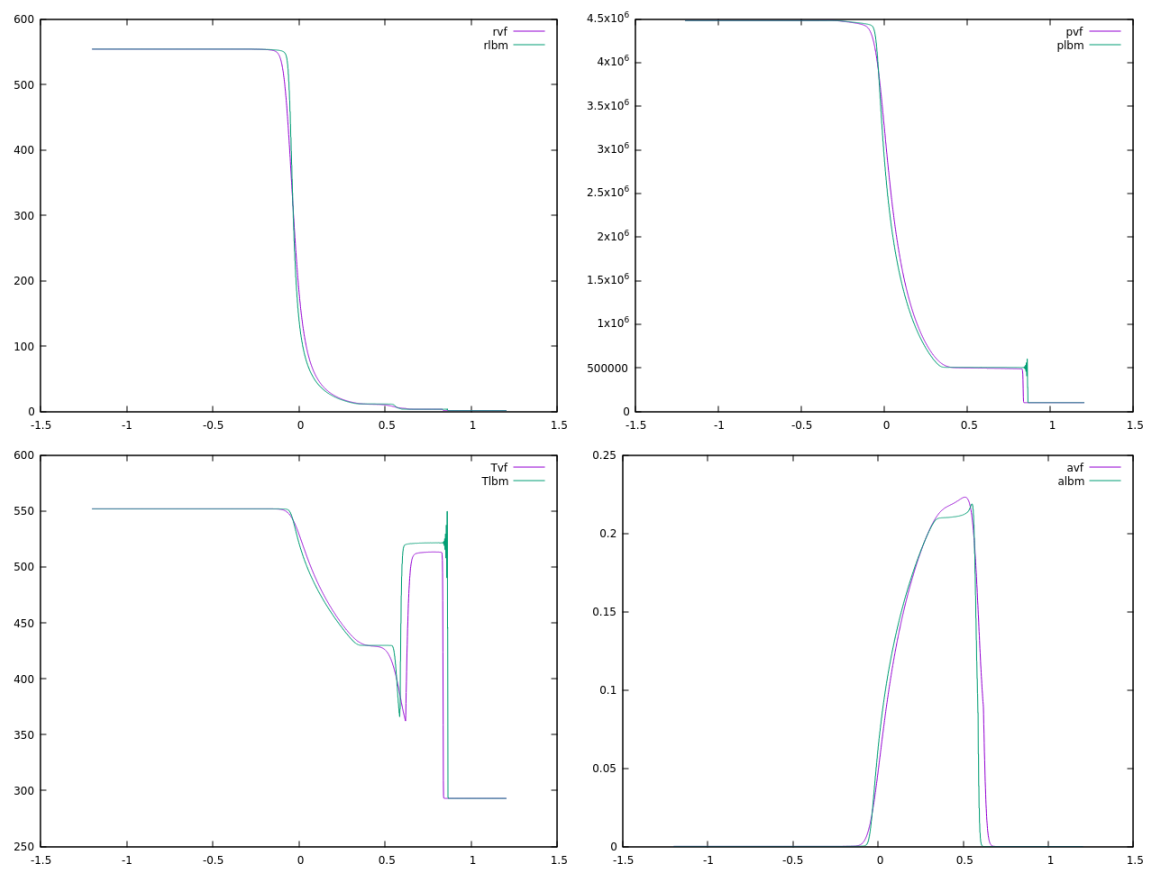

Fig. 1 Numerical solution of the Riemann problem described in Table ??. Top left: density, top right: pressure, bottom left: temperature, bottom right: vapour mass fraction. Comparison between the Finite Volume and Lattice Boltzmann Method with $\Omega=1.9 \mathrm{I}$ on a mesh with 2000 cells.

On Figure 1, we compare the numerical solutions obtained by the FV and the LBM schemes. The LBM is tested with an over-relaxation parameter $\Omega=\mathbf{I}$ (first order) and $\Omega=1.9$ I (improved precision). The second order LBM scheme with $\Omega=2$ I is unstable here, which is not surprising because there is a shock wave to capture. The results of the LBM scheme with $\Omega=\mathbf{I}$ are not plotted because they 
150 are almost superimposed with the results of the first order FV scheme. We observe a better precision of the improved LBM scheme with $\Omega=1.9$ I: the simple waves are better resolved. We observe small oscillations in the discontinuities. It is not surprising because $\Omega=1.9 \mathrm{I}$ corresponds almost to a second order scheme without limiters. We are currently working on a better strategy for adapting locally the value of $\Omega$ for suppressing oscillations.

\section{Conclusion}

We have constructed a numerical scheme based on the LBM. This scheme is faster and more precise than a classical FV method. It has been successfully validated on a complex three-phase flow with phase transition. It is possible to adjust its precision and stability thanks to the over-relaxation parameter $\Omega$, with no additional computational cost. In future works we will study strategies for completely avoiding numerical oscillations in shock waves. This can certainly be achieved because the LBM scheme with $\Omega=\mathbf{I}$ is free of oscillations and entropy-dissipative.

\section{References}

1. Mathieu Bachmann, Siegfried Müller, Philippe Helluy, and Hélène Mathis. A simple model for cavitation with non-condensable gases. In Hyperbolic Problems: Theory, Numerics and Applications (In 2 Volumes), pages 289-296. World Scientific, 2012.

2. François Bouchut. Construction of BGK models with a family of kinetic entropies for a given system of conservation laws. Journal of Statistical Physics, 95(1-2):113-170, 1999.

3. David Coulette, Emmanuel Franck, Philippe Helluy, Michel Mehrenberger, and Laurent Navoret. High-order implicit palindromic discontinuous galerkin method for kinetic-relaxation approximation. Computers \& Fluids, 190:485 - 502, 2019.

4. Clémentine Courtès, David Coulette, Emmanuel Franck, and Laurent Navoret. Vectorial kinetic relaxation model with central velocity. application to implicit relaxations schemes. 2018.

5. Florence Drui, Emmanuel Franck, Philippe Helluy, and Laurent Navoret. An analysis of overrelaxation in a kinetic approximation of systems of conservation laws. Comptes Rendus Mécanique, 347(3):259-269, 2019.

6. François Dubois. Equivalent partial differential equations of a lattice boltzmann scheme. Computers \& Mathematics with Applications, 55(7):1441-1449, 2008.

7. Amiram Harten, Peter D Lax, and Bram van Leer. On upstream differencing and godunov-type schemes for hyperbolic conservation laws. SIAM review, 25(1):35-61, 1983.

8. Philippe Helluy and Hélène Mathis. Pressure laws and fast legendre transform. Mathematical Models and Methods in Applied Sciences, 21(04):745-775, 2011.

9. Shi Jin and Zhouping Xin. The relaxation schemes for systems of conservation laws in arbitrary space dimensions. Communications on Pure and Applied Mathematics, 48(3):235-276, 1995.

10. Hiroshi Otomo, Bruce M Boghosian, and François Dubois. Two complementary latticeboltzmann-based analyses for nonlinear systems. Physica A: Statistical Mechanics and its Applications, 486:1000-1011, 2017. 